Note

\title{
The Development of a Novel, Validated, Rapid and Simple Method for the Detection of Sarcocystis fayeri in Horse Meat in the Sanitary Control Setting
}

\author{
MASATO FURUKAWA ${ }^{1,2}$, YASUTAKA MINEGISHI ${ }^{3}$, SHINJI IZUMIYAMA ${ }^{4}$, \\ KENJI YAGITA ${ }^{4}$, HIDETO MORI ${ }^{5}$, TAKU UEMURA ${ }^{6}$, YOSHIKI ETOH ${ }^{7}$, ERIKO MAEDA ${ }^{7}$, \\ MARI SASAKI ${ }^{8}$, KAZUYA ICHINOSE $^{8}$, SEIYA HARADA ${ }^{1}$, YOICHI KAMATA ${ }^{9}$, \\ MASAKI OTAGIRI ${ }^{2,10}$, YOSHIKO SUGITA-KONISHI ${ }^{11}{ }^{*}$, AND TAKAHIRO OHNISHI ${ }^{12}$ \\ ${ }^{1}$ Kumamoto Prefectual Institute of Public-Health and Environmental Science, \\ 1240-1 Kurizaki-machi, Uto-shi, Kumamoto 869-0425, Japan \\ ${ }^{2}$ Faculty of Pharmaceutical Sciences, Sojo University, 4-22-1 Ikeda, Nishi-ku, Kumamoto-shi, \\ Kumamoto 860-0822, Japan \\ ${ }^{3}$ Nippon Gene Corporation, Limited Company, 1-8-7 Toiyamachi, Toyama-shi, Toyama 930-0834, Japan \\ ${ }^{4}$ Department of Parasitology, National Insutitute of Infectious Diseases, \\ 1-23-1 Toyama, Shinjuku-ku, Tokyo 162-8640, Japan \\ ${ }^{5}$ Kobe Quarantine Station, Center for Inspection of Imported Food and Infectious Diseases. \\ 1-1, Toyahamach, Hyougoku, Kobe, Japan \\ ${ }^{6}$ Kobe Institute of Health: 4-6 Minatojimanakamachi, Chuo-ku, Kobe 650-0046, Japan \\ ${ }^{7}$ Fukuoka Institute of Health and Environmental Sciences, 39 Mukaizano, Dazaifu, Fukuoka 818-0135, Japan \\ ${ }^{8}$ Oita Prefectural Institute of Health and Environment, 2-8 Takae-nishi, Oita-shi, Oita 870-1117, Japan \\ ${ }^{9}$ Faculty of Agriculture, Iwate University, 3-18-8 Ueda, Morioka-shi, Iwate 020-8550, Japan \\ ${ }^{10}$ DDS Research Institute, Sojo University, 4-22-1 Ikeda, Nishi-ku, Kumamoto-shi, Kumamoto 860-0822, Japan \\ ${ }^{11}$ Graduate School of Environmental Health Science, Azabu University, 1-17-71 Fuchinobe, Chuo-ku, \\ Sagamihara-shi, Kanagawa 252-5201, Japan \\ ${ }^{12}$ Division of Microbiology, National Institute of Health Sciences, 1-18-1 Kamiyoga, Setagaya-ku, \\ Tokyo 158-8501, Japan
}

Received 25 May, 2015/Accepted 24 September, 2015

Sarcocystis fayeri (S. fayeri) is a newly identified causative agent of foodborne disease that is associated with the consumption of raw horse meat. The testing methods prescribed by the Ministry of Health, Labour and Welfare of Japan are time consuming and require the use of expensive equipment and a high level of technical expertise. Accordingly, these methods are not suitable for use in the routine sanitary control setting to prevent outbreaks of foodborne disease. In order to solve these problems, we have developed a new, rapid and simple testing method using LAMP, which takes only 1 hour to perform and which does not involve the use of any expensive equipment or expert techniques. For the validation of this method, an inter-laboratory study was performed among 5 institutes using 10 samples infected with various concentrations of $S$. fayeri. The results of the inter-laboratory study demonstrated that our LAMP method could detect $S$. fayeri at concentrations greater than $10^{4}$ copies $/ g$. Thus, this new method could be useful in screening for $\mathbf{S}$. fayeri as a routine sanitary control procedure.

Key words : Sarcocystis fayeri / Screening / Rapid detection / LAMP.

*Corresponding author. Tel / Fax: +81-427-69-1887, E-mail :

y-konishi (a)azabu-u.ac.jp 
TABLE 1. The primer sequences in the LAMP assay for S. fayeri

\begin{tabular}{ll}
\hline \multicolumn{1}{c}{ Primer name } & \multicolumn{1}{c}{ Sequence } \\
\hline C-gADF-F3 & 5'-CCAGCACAGAACTGTACTG-3' \\
C-gADF-B3 & 5'-AAGCCGGTGAATGAACAG-3' \\
C-gADF-FIP & 5'-TCAGTCCACCTTCCTGGCTTACTGCGTGTGATGAGAAG-3' \\
C-gADF-BIP & 5'-TTGCGGTAGTGCTGATTGTGTAGACCACAGCACACTTGTT-3' \\
C-gADF-LoopF & 5'-TCCTGTTACTTCCTGTGTTCTG-3' \\
C-gADF-LoopB & 5'-AGTCACCACGCAACAGTT-3' \\
\hline
\end{tabular}

The LAMP primers were designed from the ADF intron sequence using LAMP Designer (OptiGene Inc.)

Sarcocystis fayeri (S. fayeri) is a new causative agent of foodborne diseases that is associated with the consumption of raw horse meat (Kamata et al., 2014). The incidence of $S$. fayeri food poisoning has been increasing in Japan since 2003. The incubation period of $S$. fayeri in food is short, while the main symptom of infection (in humans) is diarrhea. When outbreaks of food poisoning occur, the causative agents should be investigated. For the investigation of causative agents, the notification methods are prescribed by the Ministry of Health, Labour and Welfare of Japan (MHLW) for the detection of $S$. fayeri. The notification methods involve the combination of microscopic observation and a PCR assay (MHLW, Accessed September, 2015; Pritt et al., 2008). However, conventional PCR analysis is timeconsuming, costly and complicated, and in a typical investigation, DNA would be sampled from more than three points from meat. Furthermore, it is difficult to identify $S$. fayeri cysts by microscopic observation because they strongly resemble the marbling in muscle. As a result, the microscopic investigation requires expert technical ability. For these reasons, the notification methods are not suitable for use in the routine sanitary control setting or tests before shipment to prevent outbreaks caused by S. fayeri. We have thus developed a new rapid detection method for $S$. fayeri using loopmediated isothermal amplification (LAMP), which can be used to test multiple samples and to perform assays in the sanitary control setting.

The LAMP method is a rapid genetic screening test with high specificity that is able to amplify a small number of DNA copies to $10^{9}$ in less than an hour under isothermal conditions (Notomi et al., 2000). This method has therefore attracted a great deal of attention for its potential to provide rapid and accurate pathogen detection in both the sanitary control and clinical settings. The LAMP method has been successfully applied to the detection of microbial pathogens in food and environmental samples. It is thus considered to be useful for detecting microbial pathogens in food in the course of routine sanitary control. We herein describe our method for the detection of $S$. fayeri by a LAMP assay.

The LAMP assay protocol developed in this study was as follows. Horse meat infected with $S$. fayeri was provided by the Kumamoto Prefectural Institute of Public-Health and Environmental Science. A LAMP detection kit (Sugita-Konishi et al., 2015), consisting of EasyPrep Reagent for LAMP, Isothermal Master Mix containing a fluorescent intercalating dye, and a LAMP primer set mixture, was used. The LAMP primer for $S$. fayeri detection was designed from an ADF intron sequence by the LAMP Designer (OptiGene, Horsham, UK), as shown in Table 1.

In the actual test, a slice of the horse meat was minced and added to EasyPrep Reagent for LAMP (0.3 g/300 $\mu \mathrm{L})$. It was mixed and heated at $95^{\circ} \mathrm{C}$ for 10 minutes. After 1 minute on ice, it was centrifuged at 15,000 $\times \mathrm{g}$ for 5 minutes. A total of $100 \mu \mathrm{L}$ of the supernatant was transferred to a fresh tube and mixed with $400 \mu \mathrm{L}$ of distilled water. The LAMP assay was then carried out using a Genie ${ }^{\circledR}$ II Instrument (OptiGene, Horsham, UK). The results were analyzed in terms of $\mathrm{Tp}$ values (the time taken to generate a positive result). The reaction was performed in a final volume of $25 \mu \mathrm{L}$ containing 5.0 $\mu \mathrm{L}$ of DNA extract, $15 \mu \mathrm{L}$ of Isothermal Master Mix, 5.0 $\mu \mathrm{L}$ of LAMP primer set mixture $(1.0 \mu \mathrm{mol} / \mathrm{L}$ each of $\mathrm{F} 3$ and B3, $8.0 \mathrm{mmol} / \mathrm{L}$ each of FIP and BIP and $4.0 \mu \mathrm{mol} /$ $L$ each of Loop F and Loop B). The mixture was subsequently incubated at $66^{\circ} \mathrm{C}$ for 30 minutes with the realtime monitoring of fluorescence intensity.

Although this study was not tested with other species of the Sarcocystis genus, bacteria or with other species of parasitic insects because no base sequences have been confirmed to target the base sequences indicated by the Basic Local Alignment Search Tool (BLAST, Altschul et al., 1990), the LAMP method is considered have high specificity. Non-specific reactions to horse meat were not been detected using this method.

To validate this new method, we performed an interlab study with five institutes (A-E). A real-time PCR assay (Yagita, K., Uchida, Y., the 82nd Annual Meeting of the Japanese Society of Parasitology, 2013) was 
TABLE 2. The results of the LAMP assay validation in an inter-lab study

\begin{tabular}{ccc|cc|cc|cc|cc}
\hline Concentration* & \multicolumn{7}{c}{ Institute } \\
\cline { 2 - 4 } of copies) & $\mathrm{A}$ & $\mathrm{B}$ & $\mathrm{C}$ & $\mathrm{D}$ & $\mathrm{E}$ \\
\hline 0 & - & - & - & - & - & - & - & - & - & - \\
$1.7 \times 10^{3}$ & - & - & - & - & - & - & - & - & - & - \\
$1.0 \times 10^{4}$ & + & + & + & + & + & + & + & + & + & + \\
$3.0 \times 10^{5}$ & + & + & + & + & + & + & + & + & + & + \\
$1.2 \times 10^{6}$ & + & + & + & + & + & + & + & + & + & + \\
\hline
\end{tabular}

*The number of copies of $S$. fayeri was detected by a realtime PCR assay. We validated the LAMP assay in duplicate experiments performed at 5 institutes. "-" indicates that the assay did not detect S. fayeri; "+" indicates the detection of $S$. fayeri.

performed to estimate the concentration of $S$. fayeri in the meat used for this inter-lab study. Briefly, a slice of horse meat was minced and added to TE buffer (300 $\mathrm{mg} / \mathrm{mL}$ ). It was mixed and centrifuged at $4,200 \times \mathrm{g}$ for 5 seconds, and the supernatant was collected. The DNA extraction of the supernatant was performed with a QIAamp DNA Mini Kit (Qiagen, Hilden, Germany) according the manufacturer's instructions, with the exception of the use of $100 \mu \mathrm{L}$ of AE buffer. The Amplification of $S$. fayeri $18 \mathrm{~S}$ rRNA gene was employed with the GeneAce SYBR qPCR Mix a No ROX (Nippon Gene, Toyama, Japan) and a forward primer (1F: GAT ACA GAA CCA ATA GGG ACA TCA C) and reverse primer (3R: ACT ACC GTC GAA AGC TGA TAG G) were used under the following PCR conditions: denaturation at $95^{\circ} \mathrm{C}$ for 10 minutes; 45 cycles of $95^{\circ} \mathrm{C}$ for 30 seconds, $62^{\circ} \mathrm{C}$ for 60 seconds using a LightCycler ${ }^{\circledR} 480$ Instrument II (Roche, Rotkreuz, Switzerland).

Table 2 shows the $S$. fayeri concentrations in the horse meat samples used in the inter-lab study. Since the limit of detection of the real-time PCR assay for $S$. fayeri was $10^{3}$ copies $/ g$, the samples which were confirmed to be negative for $S$. fayeri by a conventional PCR assay were regarded as $S$. fayeri-negative. For the inter-lab study, 5 different concentrations of $S$. fayeri were chosen as blind double samples $\left(0-10^{6} \mathrm{copies} / \mathrm{g}\right)$. The collaborating laboratories were blinded to the concentrations of the 10 samples (2 samples of each concentration).

Figure 1 shows the typical chart of a LAMP assay and the results of the inter lab study in Table 2. The increase of fluorescence indicates the detection of $S$. fayeri. The samples containing $10^{3}$ copies/g of $S$. fayeri, were found to be negative by the LAMP assay at all institutes while the samples containing more than $10^{4}$ copies/g were found to be positive at all institutes. These results demonstrated that $10^{4}$ copies/g of $S$.

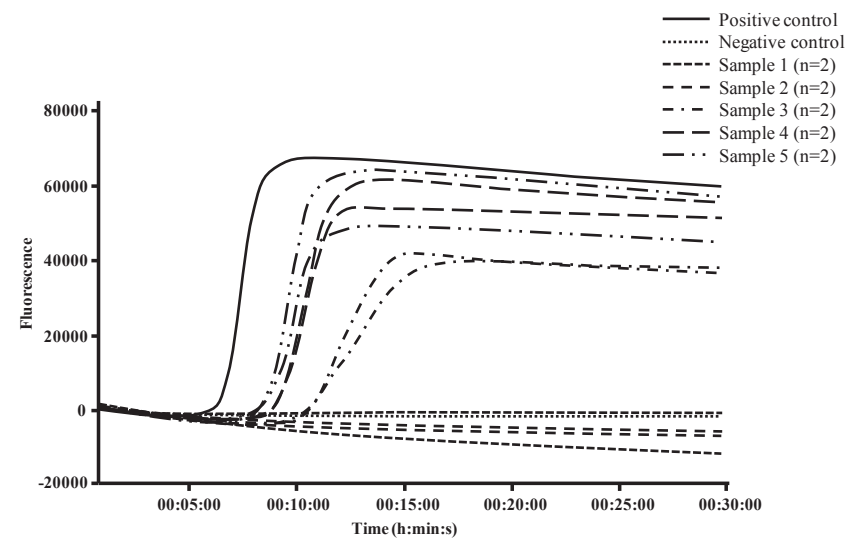

FIG. 1. The chart of the LAMP assay from institute $A$. The increase of fluorescence was considered as positive. The concentration of Sample $1(n=2)$ was 0 copy $/ \mathrm{g}$. The concentration of Sample $2(n=2)$ was $1.7 \times 10^{3}$ copies $/ g$. The concentration of Sample $3(n=2)$ was $1.0 \times 10^{4}$. The concentration of Sample $4(n=2)$ was $3.0 \times 10^{5}$ copies $/ g$. The concentration of Sample $5(n=2)$ was $1.2 \times 10^{6}$ copies $/ g$. From Sample 3, the increase was detected.

fayeri was the limit of detection by the LAMP assay and that this method was able to consistently detect $S$. fayeri in meat at concentrations of greater than $10^{4}$ copies/g.

From a performance aspect, the new LAMP method has some advantages over the notification method. The first is in the time required to perform the LAMP assay: the entire procedure, from the extraction of DNA to the end of the analysis, can be performed within 1 hour. In contrast, it takes two to three hours to perform a PCR assay using the conventional method. Thus the LAMP method is considerably faster. The next advantage is in its implementation. The LAMP method involves few steps and no complicated handling procedures. Sanitary control procedures aim to prevent outbreaks. Any detection system that is designed for application in a sanitary control setting should be easy to apply and be capable of providing quick results. Usually, the horse meat that is used for detection purposes has a great deal of marbling. It is therefore quite difficult to detect $S$. fayeri in the meat by microscopy because $S$. fayeri cysts are likely to be contained within the marbling of the muscle. This new kit allows for $S$. fayeri to be easily detected without the need for complicated procedures. Thus, even staff members without experience in $S$. fayeri detection would be able to successfully detect $S$. fayeri.

A PCR analysis (using the conventional method) of specimens from two $S$. fayeri outbreaks, which occurred in Kumamoto after October 2011-January 2012, revealed copy numbers of $10^{7}$ copies/g and $10^{6}$ copies/g. Despite the limited number of outbreaks from which we can investigate real-time PCR results, the findings provide 
good evidence for the estimation of the threshold level for $S$. fayeri-related illness to be $\geq 10^{6}$ copies $/ g$. From this, it seems that more than $10^{6}$ copies/g of $S$. fayeri might be needed for the onset of illness.

It is generally accepted that the maximum level of a foodborne hazard for enforcement or food sanitary control should be set, according to principle, to be as low as reasonably achievable (ALARA). Since S. fayeri does not grow in meat and it seems that there is a concentration level to trigger illness, this could be considered as a chemical hazard. The limit of detection of this new method was $10^{4}$ copies $/ \mathrm{g}$, which was $1 / 100$ of the value of the estimation of the threshold level for illness Thus, this limit of detection is considered to be reasonable in a screening assay for daily sanitary control and for investigations to detect causative agents in food poisoning.

In conclusion, we validated the performance of a new method of LAMP assay in the detection of $S$. fayeri. This new method is simple to perform and provides rapid results. The new LAMP method could be especially useful in the sanitary control setting, where it is necessary to quickly test a large number of samples. Because this new method satisfies these conditions, we suggest that it is suitable for testing multiple samples and for the rapid and correct detection of $S$. fayeri in horse meat samples in a sanitary control setting.

\section{REFERENCE}

Altschul, S. F., Gish, W., Miller, W., Myers, E, W., and Lipman, D. J. (1990) Basic local alignment search tool. J. Mol. Biol., 215, 403-410.
Compton, j. (1991) Nucleic acid sequence-based amplification. Nature, 350, 91-92.

Guatelli, J. C., Whitfield, K., M., Kwoh, D., Y., Barringer, K., J., Richman, D., D., and Gingeras, T. R. (1990) Isothermal, in vitro amplification of nucleic acids by a multienzyme reaction modeled after retroviral replication. Proc. Natl. Acad. Sci. USA, 87, 1874-1878.

Harada, S., Furukawa, M., Tokuoka, E., Matsumoto, K., Yahiro, S., Miyasaka, J., Saito, M., Kamata, Y., Watanabe, M., Irikura, D., Matsumoto, H., and Sugita-Konishi, Y. (2013) Control of Sarcocystis fayeri in horsemeat by freezing treatment and prevention of food poisoning caused by raw consumption of horsemeat. Shokuhin Eiseigaku Zasshi (in Japanese), 54, 198-203.

Kamata, Y., Saito, M., Irikura, D., Yahata, Y., Ohnishi, T., Inui, T., Watanabe, M., and Sugita-Konishi, Y. (2014) A toxin isolated from Sarcocystis fayeri in raw horsemeat may be responsible for food poisoning. J. Food Prot., 77, 814-819.

MHLW, (Accessed September 2015) Notification method. http://www.mhlw.go.jp/topics/bukyoku/iyaku/syoku-anzen/ gyousei/dl/111115_01.pdf.

Notomi, T., Okayama, H., Masubuchi, H., Yonekawa, T., Watanabe, K., Amino, N., and Hase, T. (2000) Loopmediated isothermal amplification of DNA. Nucleic Acids Research, 28, e63.

Pritt, B., Trainer, T., Simmons-Amold, L., Evans, M., Dunams, D., and Rosenthal, B., M. (2008) Detection of sarcocystis parasites in retail beef: a regional survey combing histological and genetic detection methods. J. Food Prot., 71, 21442147.

Sugita-Konishi, Y., Fukuda, Y., Mori, K., Mekata, T., Namba, T., Kuroda, M., Yamazaki, A., and Ohnishi, T. (2015) New Validated rapid screening methods for identifying Kudoa septempunctata in olive flounder (Paralichtys olivaceus). Jpn. J. Infect. Dis., 68, 145-147.

Walker, G., T., Fraiser, M., S., Schram, J., L., Little, M., C., Nadeau, J., G., and Malinowski, D., P. (1992) Strand displacement amplification-an isothermal, in vitro DNA amplification technique. Nucleic Acides Res., 20, 1691-1696. 